\title{
Computer Model for Determining Localized Changes in Ventricle Wall Thickness as a Function of Changing Wall Stress Determined by Laplace's Law
}

\author{
Richard L Summers \\ University of Mississippi Medical Center, Jackson, Mississippi, USA
}

\begin{abstract}
The ventricular myocardium remodels in an attempt to normalize local wall stress $(\sigma)$ created by physical forces and optimize cardiac function. This $\sigma$ is considered a major driving determinant of growth or regression of myocardial cells that in turn change the local ventricular myocardial wall thickness $(h)$ and area stress. A computer model was developed that describes the magnitude and time course of changes in $h$ in response to variations in physical factors (pressure, geometry). The model algorithm describes the driving changes in myocardial protein synthesis and $h$ based on a stimulus of wall stress $\left(\Delta_{s}\right)$ outside of the normal range $\left(\sigma_{o}\right)$. Changes in the segmental wall thickness (dh/dt) are considered directly related to the differential in $\sigma$ integrated over time. As $h$ changes, the actual wall stress $\left(\sigma_{a}\right)$ also changes as defined by Laplace's Law. The iteratively determined $\sigma$ drives the growth/atrophy of the ventricle wall until an equilibrium is reached and $\Delta_{s}=0$. Model performance was compared to values from the literature that examine changes in $\sigma_{a}$ during a clinical perturbation and was found to closely map changes in $\sigma_{a}$ with an exponential approach to a maximum loss of about $35 \%$ of total $h$ at 60 days with a $k$ value of 0.0006 . This model can serve as a driving algorithm for defining myocardial plasticity in a FEM of the heart and used to study cardiac remodeling.
\end{abstract}

\section{Introduction}

It has been conventional wisdom that the ventricular myocardium hypertrophies or atrophies in an attempt to normalize wall stress and thereby optimize cardiac function $[1,2]$ The degree of stress within the wall of the heart is commonly considered a major determinant of the growth or regression of myocardial cells that change the wall thickness [1-3]. A hypertrophied ventricle (thickened wall) reduces wall stress. Hypertrophy can be thought of as a mechanism that permits more muscle fibers (actually, sarcomere units) to share in the wall tension that is determined at a given pressure and radius. The thicker the wall, the less sarcomere unit tension.

\section{Methods}

A computer model was constructed using VisSim ${ }^{\mathrm{TM}}$, a graphical language for simulation and model-based embedded development, which incorporates an algorithm that describes myocardial muscle fiber growth or regression based on local wall stress.

\subsection{Algorithm development}

An algorithm that describes a process for driving changes in the wall thickness was developed and is outlined below. In this algorithm, changes in wall stress $\left(\Delta_{\mathrm{s}}\right)$ outside of the normal range $\left(\sigma_{0}\right)$ provide a stimulus that drives an increase in myocardial cellular protein synthesis and an increase in cell diameter and wall thickness (h). The change in the thickness in the wall $(\mathrm{dh} / \mathrm{dt})$ is considered directly related to the differential in wall stress from the normal value. The changing wall thickness is integrated over time. Since this process occurs at the cellular level it is expected that the changes in ventricle wall thickness also occurs segmentally at a localized level of the myocardium. As the local wall thickness changes, it is expected to change the actual wall stress $\left(\sigma_{a}\right)$ in an inverse manner as defined by Laplace's Law. This new wall stress continues to drive the growth/atrophy of the ventricle wall until an equilibrium is reached and $\Delta_{\mathrm{s}}=0$.

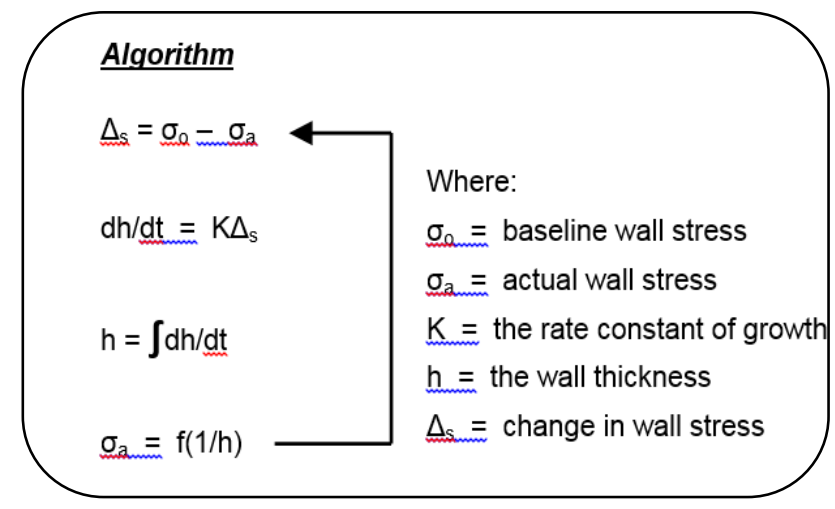




\section{$2.2 \quad$ Derivation of $\mathbf{K}$}

The value of $\mathrm{K}$ is derived from the information in the literature that analyzes changes in wall stress seen during some clinical perturbation (blood pressure medications, heart surgery) [3-6]. There is insufficient information describing these changes during spaceflight particularly as it relates to a longitudinal time course. The amount of absolute loss in wall thickness after an intervention maps the degree of change in wall stress. We would expect that descriptions that include the earliest portions of the time courses to best indicate the rate of atrophy. The best complete time course data incorporating both short and long-term changes is from Madigan et al [4]. In this study, an LVAD was used to suddenly reduce the intraventricular pressures in patients with hypertrophy due to heart failure while changes in myocyte diameter were followed over a period of 160 days. There appears to be an exponential relationship in the loss as it approaches its maximum. This information was used to calculate a $\mathrm{k}$ value using a computer model incorporating the algorithm and average data from the study (figure below). As the mass moves from $100 \%$ to $0 \%$ of the total myocyte diameter loss (maximum amount loss is about $35 \%$ of the thickness) it takes approximately 60 days. Using our model, the $\mathrm{k}$ value is 0.0006 best fits the 60 day timeframe for equilibrium. This is also consistent with the timeframe for ventricular muscle hypertrophy/atrophy to create an equilibrium in wall stress as determined by other researchers $[3,6]$.

\section{Results}

The model performance was compared to values derived from the literature that examine changes in $\sigma_{\mathrm{a}}$ during some clinical perturbation. The absolute loss in $\mathrm{h}$ after intervention was found to closely map changes in $\sigma_{\mathrm{a}}$ as predicted by the model with an exponential approach to a maximum. The maximum loss is about $35 \%$ of total $h$ at 60 days with a $\mathrm{k}$ value of 0.0006 that best fits the timeframe for equilibrium. (computer simulation below)

\subsection{Limits of atrophy/hypertrophy}

The myocardium is comprised of many elements of which the contractile proteins are only a portion. Cardiac plasticity involves only those tissue elements that change in response to variations in the wall stress. Some of the solid elements such as collagens and organelles are not directly impacted by the atrophy/hypertrophy process. The limits in both hypertrophy and atrophy have been described by several investigators [7-9]. The lower limit of atrophy of myocardial mass and hence the wall thickness is a loss of about 35\%. The upper limit of hypertrophy of these same structures is an increase of about 50\%. (graphic below derived from Madigan [4]). The computer model and simulation was found to accurately map these changes.

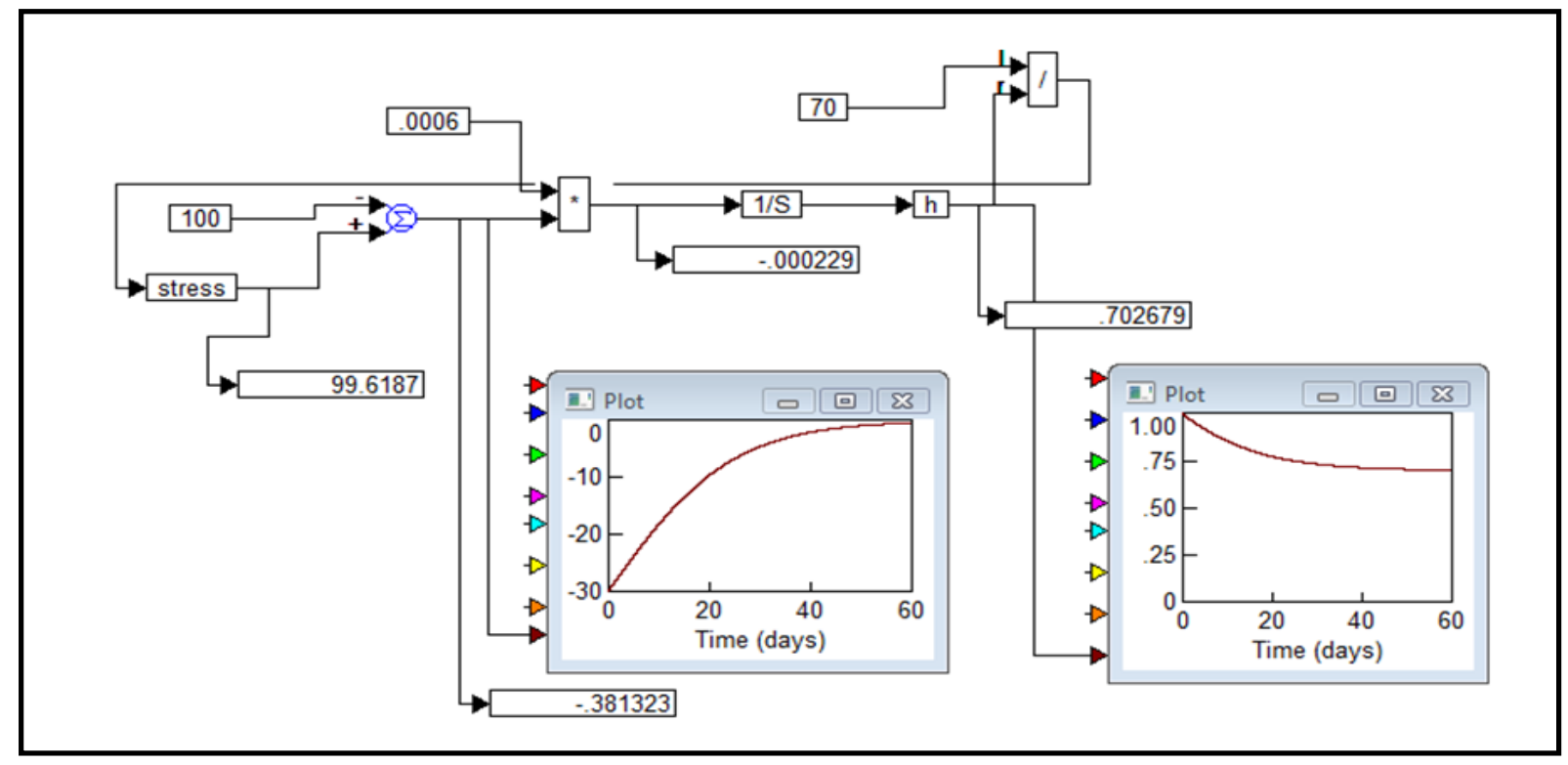

Graphical Representation of Simulation 


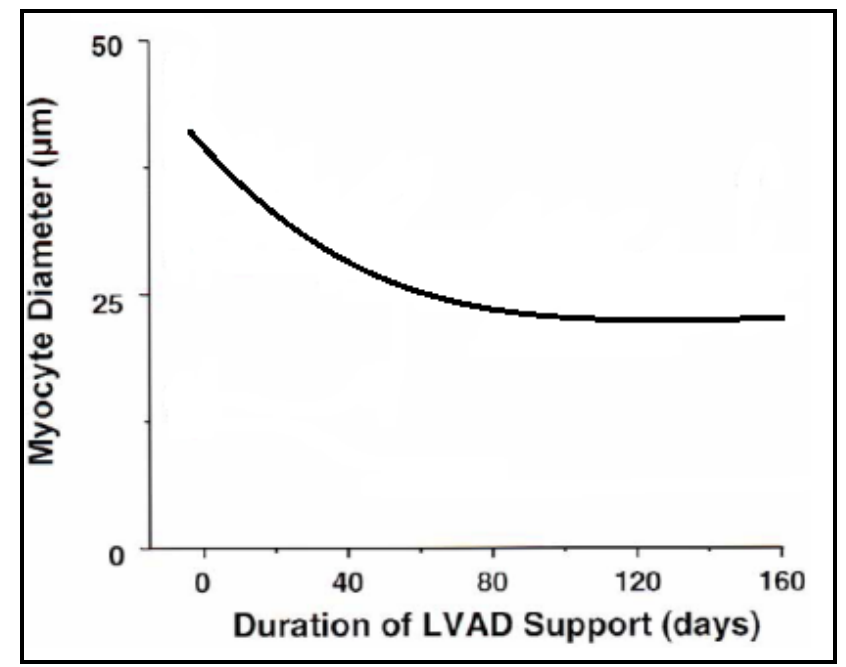

\section{Derived from Madigan et al. (reference 4)}

\section{Conclusion}

The mechanism driving the observed myocardial remodeling in astronaut hearts has been poorly understood. It has been suggested that the discerned increases in cardiac sphericity during microgravity exposure could result in reduction in wall stress in localized areas of the myocardium through the mechanism of a change in the radius of curvature as described by Laplace's law [10]. However, the direct causal relationship between these cardiac shape changes and the observed remodeling is much too difficult to directly examine experimentally. A theoretical causal relationship is currently being investigated using a FEM model of the heart [11]. While this model has clearly demonstrated the direct causal impact of microgravity on cardiac shape changes, the next step of determining if these simulations can also reproduce the cardiac remodeling seen in astronauts has yet to be accomplished. The results presented in this current study suggest that this model can serve as a driving algorithm for defining myocardial plasticity in a FEM of the heart and can be used to examine the effect of various conditions on cardiac remodeling. Such a model will be used to further investigate the theoretical consistency of our hypothesis concerning the impact of prolonged microgravity exposure during spaceflight on myocardial remodeling in the human heart $[10,11]$.

\section{References}

[1] Abi-Samra F, Fouad FM, and Tarazi RC. Determinants of left ventricular hypertrophy and function in hypertensive patients. An echocardiographic study. Am J Med 1983;75:26-33.

[2] Badeer HS. The stimulus to hypertrophy of the myocardium. Circulation. 1964;30:128-36.

[3] Ishihara K, Zile MR, Tomita M, Tanaka R, Kanazawa S, Carabello BA. Left ventricular hypertrophy in a canine model of reversible pressure overload. Cardiovasc Res. 1992;26(6):580-5.

[4] Madigan JD, Barbone A, Choudhri AF, Morales DL, Cai B, $\mathrm{Oz}$ MC, Burkhoff D. Time course of reverse remodeling of the left ventricle during support with a left ventricular assist device. J Thorac Cardiovasc Surg. 2001;121(5):902-8.

[5] Gelsomino S, Frassani R, Morocutti G, Nucifora R, Da Col P, Minen G, Morelli A, Livi U. Time course of left ventricular remodeling after stentless aortic valve replacement. Am Heart J. 2001;142(3):556-62.

[6] Mirsky I, Laks MM. Time course of changes in the mechanical properties of the canine right and left ventricles during hypertrophy caused by pressure overload. Circ Res. 1980;46(4):530-42.

[7] Hill JA, Olson EN. Cardiac plasticity. N Engl J Med. 2008;358(13):1370-80.

[8] de Groot PC, van Dijk A, Dijk E, Hopman MT. Preserved cardiac function after chronic spinal cord injury. Arch Phys Med Rehabil. 2006;87(9):1195-200.

[9] Katsume H, Furukawa K, Azuma A, Nakamura T, Matsubara K, Ohnishi K, Sugihara H, Asayama J, Nakagawa M. Disuse atrophy of the left ventricle in chronically bedridden elderly people. Jpn Circ J. 1992;56(3):201-6.

[10] Summers RL, Martin DS, SH Platts, Mercado-Young R, Coleman TG, Kassemi M. Ventricular Chamber Sphericity During Spaceflight and Parabolic Flight Intervals of Less Than 1 G. Aviat Space Environ Med 2010;81:506-510.

[11] Summers RL, Smith W, Gilbrech R, Liao J, Weed BC, Patnaik S. Impact of the Microgravity Environment on Left Ventricular Sphericity in a FEM of the Heart. 42nd International Conference on Environmental Systems. Session: ICES513: Computational Modeling for Human Health and Performance Analysis. Proceedings 2012. AIAA 2012-3448.

Address for correspondence.

Richard L. Summers, MD.

University of Mississippi Medical Center. 2500 North State Street, Jackson MS 39216, USA rsummers@umc.edu 
